\title{
Conservation Des Ressources Naturelles Pour Une Sécurité Alimentaire Durable Au Maroc
}

\author{
Ibtissam Motib, \\ Mouhcine Batchi, \\ Faycal Fatah,
}

Université Ibn Tofail, Kenitra, Faculté des lettres et des sciences humaines

Département de Géographie, Laboratoire Environnement,

Développement et Gestion de l'espace

\section{Résumé}

$\mathrm{Au}$ Maroc, la question de la conservation des ressources naturelles se pose avec autant d'importance et d'acuité, puisqu'elle touche le droit de l'homme, à l'alimentation, à la soutenabilité économique et à la durabilité environnementale. Pour produire de la nourriture, il est important d'avoir des terres cultivables et des ressources en eau suffisantes. Il faut que ces ressources soient maintenues dans la durée pour assurer l'équité intergénérationnelle. Mais face aux multiples pressions qui pèsent sur ces ressources, la dégradation de ces derniers, prend une ampleur de plus en plus préoccupant eau Maroc. Dans cet article, on se propose d'éclairer le lecteur d'une part, sur l'état des ressources naturelles au Maroc, et d'autre part de démontrer le rôle du droit comme outil ou moyen de protection de ces ressources afin de garantir la sécurité alimentaire.

Mots clés: Maroc, Sécurité alimentaire, Valorisation des ressources naturelles, Analyse économique, Approche juridique 


\title{
Conservation of Natural Resources for Sustainable Food Security in Morocco
}

\author{
Ibtissam Motib, \\ Mouhcine Batchi, \\ Faycal Fatah,
}

Université Ibn Tofail, Kenitra, Faculté des lettres et des sciences humaines

Département de Géographie, Laboratoire Environnement,

Développement et Gestion de l'espace

\begin{abstract}
In Morocco, the question of the conservation of natural resources arises with as much importance and acuity, since it touches the human right to food, economic sustainability and environmental durability. To produce food, it is important to have arable land, sufficient water resources, etc. These resources must be maintained over time to ensure intergenerational equity. However, faced with the multiple pressures weighing on these resources, the degradation of natural resources in Morocco is taking on an increasingly worrying scale. In this article, we propose to shed light on the one hand, on the state of natural resources in Morocco, and on the other hand to demonstrate the role of law as a tool or a means of protecting natural resources in order to guarantee food security.
\end{abstract}

Keywords: Morocco, Food security, Valorization of natural resources, Economic analysis, Legal approach

\section{Introduction}

Il est un fait certain qu'assurer à tous les êtres humains, à tout moment, un accès physique et économique à une nourriture suffisante et saine avec une variété alimentaire du choix des consommateurs, devient un défi crucial dans le monde (Oliver, 2014). Ce défi, serait plus difficile à relever en raison des changements climatiques, qui réduisent la productivité de la majorité des systèmes de production existants et compromet les moyens d'existence des populations, déjà exposées à l'insécurité alimentaire (HLPE, 2012).

Les États sont de plus en plus conscients de l'urgente nécessité de travailler pour atteindre l'objectif de la sécurité alimentaire aux niveaux international et national. De nombreux états (Olivier, 2014), y compris le 
Maroc, cherchent les méthodes adéquates en vue de lutter contre l'insécurité alimentaire.

L'eau et le sol sont les deux principales ressources nécessaires à l'agriculture et à la vie. Quand l'eau est abondante et les sols sont fertiles, les populations peuvent subsister, à condition que le climat soit lui aussi favorable. Mais quand l'eau vient à manquer, l'agriculture dépérit et l'insécurité alimentaire règne. L'explosion démographique et les besoins alimentaires qui en suivent, il est de plus en plus difficile de mettre davantage d'eau à la disposition des agriculteurs (FAO, 1996). Etant donné les pressions croissantes qui s'exercent sur elles, il est plus essentiel et plus urgent que jamais de bien gérer les fragiles ressources en terre et en eau de la planète (FAO, 1996).

Si l'on veut répondre aux besoins d'une population qui ne cesse de s'accroitre, il faut préserver et reconstituer ces ressources naturelles. L'exploitation de la terre doit se faire uniquement après une évaluation scientifique de la capacité des sols, l'épuisement annuel de la couche arable. Pour la réutilisation des ressources en eau, les agriculteurs sont amenés notamment à adopter de bonnes méthodes d'exploitation et de gestion, d'où vient le rôle de la discipline juridique.

L'objectif de cette étude est d'évaluer la gestion et la protection des ressources naturelles alimentaires sur le plan juridique au Maroc.

\section{Matériel et méthodes}

\section{Méthodologie du travail}

\section{Modèle « état-pression-réponse (EPR) » pour l'analyse diagnostic}

La nature complexe de la gestion des ressources naturelles exige d'adopter un outil de structuration des informations, à ce titre, nous avons opté pour le modèle EPR « pression-état -réponse », comme «boîte à outil » ce modèle, préconisé par l'OCDE (1998), ce modèle est utilisé pour décrire les types de données suivis par le dispositif de collecte, repose en effet sur la notion de causalité: les activités humaines exercent des pressions sur l'environnement et modifient la qualité et la quantité des ressources naturelles (Roudier Jean, 2004). La société répond à ces changements en adoptant des mesures de gestion et de protection qui ont pour objectifs de prévenir, réduire ou compenser les pressions et parvenir aux états souhaités. La mise en œuvre de la trame «PER » nécessite une base d'information quantitatives et qualitatives qui permettent d'établir des corrélations entre « pression » et « état » afin de surveiller le devenir du second vis-à-vis des objectifs de gestion et prévoir comment un changement de gestion modifie les pressions, et donc, en retour, l'état (Roudier Jean, 2004).

$\mathrm{Si}$ «l'état»» indique la situation des ressources naturelles, la «pression » exprime les activités humaines engendrant les menaces sur les 
ressources, tandis que la « réponse » constitue les mesures prises pour atténuer les impacts sur le support naturel. En matière de non-réponse, on peut évoquer l'absence de système de gestion des ressources naturelles (Mélanie, 2012).

\section{Approche juridique}

Ce travail de recherche est également influencé par l'approche juridique en matière de respect des ressources naturelles. En effet le droit, diton, est un outil de diagnostic. Il permet d'analyser un phénomène dans sa profondeur (Motib, 2019). L'important pour lui, c'est de découvrir les causes et de proposer des solutions. Certes, il est en mesure de définir des méthodes simples et modestes susceptibles d'apporter des grandes solutions aux problèmes de surexploitation des ressources naturelles. Il cherche toujours à segmenter les problèmes systémiques, ce qui rend son travail d'analyse plus facile et son pouvoir d'agir plus efficace. De là, nous utilisons le droit comme un outil de diagnostic et de méthodologie. Bien évidemment, l'enjeu de la gestion des ressources naturelles alimentaires à trouver un équilibre entre utilisation durable et protection, notamment en mettant l'usage au cœur de la démarche et des réflexions.

\section{Ressources naturelles au Maroc Etats des lieux et diagnostic}

De tout temps, l'homme entretien avec la nature plusieurs types de rapports qui lui permettent d'assurer sa survie et son épanouissement. Ainsi les populations et leurs activités influencent l'environnement par leurs effectifs, leurs densités et leurs croissances, en d'autre terme, « la densité de la population est le meilleur critère de plus ou moins grande intensité de l'exploitation » (Gourou, 1940). C'est ainsi que la gestion des ressources naturelles (OMC, 2010) a toujours été une préoccupation pour les groupes sociaux et les pouvoirs publics.

Cette question requiert une importance vitale pour le Maroc puisqu'il connait une croissance démographique continue. Par conséquent, pour la satisfaction de leurs besoins, la pression sur l'environnement prend de plus en plus d'ampleur. En effet l'homme met ainsi en péril la biodiversité pour ses besoins alimentaires. Or, malgré les efforts consentis en matière de gestion conservatoire des ressources naturelles, le diagnostic des systèmes d'exploitation de ces ressources, relève qu'elles ont plus de mal à se renouveler pour assurer leur pérennité (Abdellah, 2016). Cette situation factuelle révèle qu'il s'agit là d'un des principaux défis auquel le Maroc doit faire face dans les années à venir pour assurer sa sécurité alimentaire. 


\section{L'eau: une ressource de plus en plus menacée}

Aujourd'hui, la question de l'eau se pose avec acuité au Maroc. Le pays est classé parmi les 20 pays les plus stressés en termes de disponibilité des ressources en eau. Il est indexé à 4,2 sur une échelle de 5, le qualifiant comme un pays « extrêmement risqué » en matière de disponibilité en eau (Abdellatif et Lelia, 2017). Cette vulnérabilité est d'autant plus importante que la sécurité alimentaire du pays dépend de l'agriculture, elle-même fortement liée à la pluviométrie et à la disponibilité des ressources hydriques (WRI, 2014)

\section{Eaux de surfaces}

De par sa situation géographique, le Maroc est caractérisé par un climat à la fois méditerranéen au nord et aride au sud et au sud-est de l'Atlas, avec une saison sèche et chaude et une saison froide et humide. Il bénéficie de nombreux atouts naturels; des pluies d'ouest réassez importantes, des rivières pérennes comme le Sebou ou l'Oum er Ria, d'importantes nappes souterraines, même dans les régions méridionales (SCN, 2016).

Les eaux de surface sont assurées principalement par135 grands barrages, elles sont évaluées en année moyenne à 18 milliards de $\mathrm{m}^{3}$, variant selon les années de 5 milliards de $\mathrm{m}^{3}$ à 50 milliards de $\mathrm{m}^{3}$ (Ministère de l'équipement, et de l'eau, 2016).

Le régime hydrologique de l'ensemble des bassins est caractérisé par une très grande variabilité interannuelle marquée par l'alternance des séquences humides et sèches, intercalées par des années de forte hydraulicité ou de sécheresse sévère (RFB, 2019).

La grande disparité régionale des précipitations induit également une grande variabilité spatiale des écoulements d'eau de surface. Ces derniers varient de quelques millions de $\mathrm{m}^{3}$ pour les bassins les plus arides, tels que les bassins Sahariens, du Souss-Massa-Tiznit-Ifni, du ZI, Rhéris, Guir, Bouâanane et Maïder à des milliards de $\mathrm{m}^{3}$ par an pour les bassins les plus arrosés, tels les bassins du Loukkos, du Tangérois, des Côtiers Méditerranéens et $\mathrm{du}$ Sebou. Les bassins du nord (Loukkos, Tangérois et Côtiers méditerranéens) et le Sebou qui couvrent près de $7 \%$ de la superficie du pays disposant de plus de la moitié des ressources en eau (RBF, 2019).

\section{Eaux souterraines}

Les eaux souterraines représentent environ $20 \%$ du potentiel en ressources en eau du pays. Sur les 103 nappes répertoriées, 21 sont des nappes profondes et 82 superficielles. Les plus importants systèmes aquifères couvrent une superficie totale de près de $80000 \mathrm{~km}^{2}$, soit environ $10 \% \mathrm{du}$ territoire. A l'état actuel des connaissances, le potentiel en eau souterraine, est de l'ordre de 4,3 Milliards de $\mathrm{m}^{3}$ par an (Abdellatif et Lelia , 2017). Cette 
estimation reste tributaire du niveau d'évaporation des eaux et de l'intensité des précipitations qui dépendent du changement climatique. Le stockage des eaux souterraines représente une ressource d'importance stratégique pour faire face aux sècheresses futures.

Les eaux souterraines constituent également la principale ressource pour l'approvisionnement en eau potable des populations rurales et contribuent à la sécurisation de plusieurs villes du pays. Par ailleurs, cette ressource stratégique a permis le développement de l'irrigation privée sur une superficie de 570000 ha, axée sur des spéculations agricoles très valorisantes de l'eau et à forte valeur ajoutée destinée principalement à l'exploitation (près de $54 \%$ de la valeur ajoutée). Cependant, malgré leur caractère stratégique, ces ressources sont gérées d'une manière non durable. En fait, le suivi de l'évolution des niveaux d'eau de la quasi-totalité des nappes du pays, montre une baisse continue atteignant parfois des valeurs alarmantes pouvant $2 \mathrm{~m} / \mathrm{an}$ (Ministère de l'énergie, des mines et de l'environnement, département de l'environnement, 2016).

Les apports pluviométriques se caractérisent par une forte irrégularité spatiale et temporelle. Environ 50\% de ces apports sont concentrés sur 15\% de la superficie totale du pays. Par ailleurs, 67\% des ressources en eaux superficielles sont stockées dans les bassins hydrauliques de Louksor, Sebou et d'Oum Er Ria.

Il est à noter que, les ressources en eau au Maroc sont parmi les plus faibles au monde (Benmalek, 2017). En effet, le potentiel des ressources en eau naturelles, est évalué à 22 milliards de $\mathrm{m}^{3}$ par an, soit l'équivalent de 730 $\mathrm{m}^{3} /$ habitant/an, Communément admis comme seuil critique indiquant l'apparition de pénuries et de crise latente d'eau (World Bank, 2017). Cette quantité d'eau pourrait chuter à $510 \mathrm{~m}^{3}$ par personne d'ici 2050, ce qui est proche du «seuil de rareté extrême de l'eau» de $500 \mathrm{~m}^{3}$ par habitant (World Bank, 2017).

\section{Ressources en eau du Maroc sujettes à des pressions multiples}

Plusieurs problèmes affectent les ressources en eau au Maroc, liés à la réduction de la disponibilité et à la dégradation de la qualité de ces ressources

\section{Impact de croissance démographique sur les ressources en eaux}

Le Maroc connait un élan démographique rapide, selon les tendances dégagées par les projections démographiques réalisées par le Haut Commissariat au Plan en 2018, une variante moyenne indique que la population du Maroc passerait de 35,95 millions d'habitants en 2020 à 38 millions en 2030, ce qui équivaut à une croissance additionnelle moyenne de 300000 habitants par an, soit l'équivalent d'une grande ville. L'effectif de la population rurale restant quasi stable autour de 13,5 millions, l'évolution 
démographique marocaine serait principalement urbaine, en raison essentiellement de l'exode rural et de l'urbanisation de zones rurales. Ainsi, les villes marocaines abriteraient 64\% des habitants du pays, en 2030 (HautCommissariat au Plan, 2018).

Cette évolution démographique aura des conséquences dont les plus importantes, sont la dégradation de l'environnement et de ses ressources. La pression démographique amène aussi la réduction de la disponibilité en eau par tête d'habitant. La raréfaction de la ressource en eau risque d'être aggravée périodiquement lors des périodes de sécheresse (Abdellah, 2006).

\section{Changement climatique, la goutte qui fait déborder le vase}

Le changement climatique est aujourd'hui une réalité au Maroc, comme partout d'ailleurs dans le monde, selon le quatrième rapport d'évaluation du climat du Groupe d'experts intergouvernemental sur l'évolution du climat (GIEC, 2014). Le Maroc est très vulnérable à la variabilité du climat. Les projections climatiques établies par la direction de la météorologie nationale prévoient une augmentation des températures moyennes estivales de l'ordre de 2 à $6{ }^{\circ} \mathrm{C}$ et une régression de $20 \%$ en moyenne des précipitations d'ici la fin du siècle (Ministère délégué auprès du Ministre de l'Energie, des Mines, de l'Eau et de l'Environnement, 2014). Ce réchauffement climatique a une incidence directe sur la pluviométrie dont dépendent le taux de remplissage des barrages, la réalimentation des nappes et les eaux de surface.

Le ministère de l'Environnement, dans une étude intitulée «Politique du changement climatique au Maroc » en 2014, prévoit une baisse significative des précipitations oscillant entre 3 et $30 \%$ selon les régions; une accélération des phénomènes extrêmes (notamment les sécheresses et les inondations), une tendance à la hausse des vagues de chaleur et la baisse des vagues de froid et une élévation du niveau de la mer (Ministère délégué auprès du Ministre de l'Energie, des Mines, de l'Eau et de l'Environnement, 2014). L'Institut royal des études stratégiques rappelle dans son rapport « le Maroc face au changement climatique. Incidences sur la trajectoire de développement et perspectives d'adaptation " que les sécheresses, qui représentent un trait structurel de notre climat, sont devenues particulièrement aiguës et fréquentes. Le Maroc est passé d'une sécheresse tous les dix ans durant la période de 1950 à 1960et de deux à trois sécheresses par décennie depuis les années 1980. Durant la période de 1955 à 2004, le Maroc a dû faire face à 7 périodes de sécheresse généralisée, dont 5 après 1975 (L'Institut royal des études stratégiques, 2011).

À la fin du siècle, les projections font état d'une baisse des précipitations d'environ $20 \%$ et qu'elle atteindrait $40 \%$ dans les zones situées à l'ouest de la chaine de l'Atlas, il en ressort qu'à la fin du siècle, les 
projections font état d'une baisse des précipitations d'environ $20 \%$ et qu'elle atteindrait 40\% dans les zones situées à l'ouest de la chaine de l'Atlas. Par ailleurs, le barrage Idriss $1^{\mathrm{er}}$ sur l'oued Inaouene (bassin du Sebou) verrait son débit, chuter de 7,6\% à l'horizon 2080 (selon le scénario optimiste) et de $40 \%$ (scénario pessimiste), (Benmalek, 2017). D’autres impacts sont également à craindre à en croire la même étude. Les changements climatiques pourraient provoquer le dérèglement des régimes naturels saisonniers des oueds, la réduction de la capacité de stockage des retenues des barrages en raison d'un envasement accéléré par une érosion accentuée des sols des bassins versants, engendrée par de fortes intensités de pluie (benmalek, 2017). Selon le secrétariat d'état auprès du Ministre de l'équipement, du transport, de la logistique et de l'eau, chargé de l'eau, la surexploitation des nappes d'eau souterraines pourrait également s'aggraver, ce qui provoquerait l'accentuation de l'approfondissement des niveaux des nappes d'eau souterraine et l'augmentation de la salinité des nappes côtières, en raison d'une invasion plus importante des eaux marines (Le Matin, 2017).

\section{Déchets causés par l'action humaine et les unités industrielles aggravent la situation}

La pollution urbaine et industrielle demeure un réel danger au Maroc, dans la plupart des centres urbains et des industries, les eaux usées sont rejetées sans traitement préalable dans le milieu récepteur comme les eaux de surface, sol, océan...etc., ce qui entraine une dégradation considérable de la qualité des ressources en eau de surface et souterraines (Haut Commissariat au Plan, 2006).

En effet, le Ministère de l'Energie, de l'Eau et de l'Environnement, a rapporté que durant la période de 2014 à 2015, les cours d'eau ont été globalement de qualité bonne à moyenne à l'exception des points de prélèvements situés en aval des rejets urbains. Par ailleurs, $71 \%$ des stations échantillonnées ont présenté une eau de qualité excellente à moyenne et de $29 \%$ des stations ont une eau de qualité mauvaise à très mauvaise (Ministère Délégué auprès du Ministère de l'Energie, de l'Eau et de l'Environnement, Chargé de l'Eau, 2015).

En ce qui concerne les eaux souterraines, la qualité bactériologique et organique des nappes contrôlées est excellente à bonne (Abdellatif et Lelia, 2017) . La qualité minéralogique et azotée a connu une détérioration par endroits ou généralisée. En effet, $45 \%$ des stations d'eaux souterraines affichent une qualité dégradée contre 55\% une qualité excellente à moyenne, résultant de la pollution par les nitrates et la salinité (Abdellatif et Lelia, 2017).

Il a été souligné que les volumes annuels des rejets des eaux usées des villes ont fortement augmenté au cours des dernières décennies. Ils sont passés de 48 à 506,2 millions de m³/an entre 1960 et 2012 (Benmalek, 2018). Près de 
$61 \%$ de ces volumes sont déversés directement dans l'océan. Le reste $(39 \%)$ est rejeté dans le réseau hydrographique ou directement dans le sol. Les prévisions établies montrent que ces rejets continueront à croître rapidement pour atteindre 741 millions de m³/an à l'horizon 2030 (Benmalek, 2018).

\section{Développement d'une agriculture grosse consommatrice d'engrais et de pesticides}

$\mathrm{Au}$ Maroc, l'agriculture dépend fortement de la pluviométrie et à la disponibilité des ressources hydriques. Les volumes d'eau consommés par l'irrigation représentent la grande partie des volumes taux consommés et atteignent $85 \%$ des ressources disponibles. Les besoins du secteur sont très conséquents du fait que $85 \%$ des terres agricoles sont encore mises en valeur en Bour ministère délégué auprès du ministère de l'énergie, des mines de l'eau et de l'environnement, 2015). La surexploitation de la ressource s'explique par le type d'irrigation pratiqué, le système gravitaire par exemple, qui est peu économe en eau.

En effet, l'activité agricole constitue une source de pollution pour la qualité des ressources en eau, du fait de la non-rationalisation des produits phytosanitaires et des engrais ainsi que les mauvaises pratiques agricoles en matière d'irrigation. Le Maroc a connu au fil de temps une augmentation des superficies irriguées. Cette évolution s'est accompagnée par la prolifération du pompage de la nappe; la nouvelle technique d'irrigation; et l'utilisation de plus en plus croissante d'engrais et des pesticides (Motib, 2019).

L'irrigation peut avoir des effets négatifs sur l'environnement, en particulier sur la qualité des eaux superficielles et souterraines à travers soit la mauvaise qualité de l'eau qu'elle applique soit en servant de vecteur aux intrants (engrais azotés et pesticides appliqués souvent en excès). Les engrais, lorsqu'ils sont appliqués en trop grande quantité par rapport aux besoins des plantes et à la capacité de rétention des sols, sont des causes majeures de la pollution de l'eau potable (liée à la toxicité des nitrates) ou de l'eutrophisation des eaux douces à travers le lessivage des éléments solubles, soit vers la nappe phréatique soit, vers les cours d'eau par ruissellement (Motib, 2019).

\section{Sol}

\section{Atout pour la sécurité alimentaire subi plusieurs pressions}

Le sol est actuellement considéré comme une interface dans l'environnement et une ressource pour le développement (Cédric, 2011). Il nourrit le monde, il produit, contient, accumule, tous les éléments nécessaires à la vie (azote, phosphore, calcium, potassium, fer, oligoéléments...). Les sociétés humaines, dépendantes totalement des sols (alimentation et santé), il est primordial donc de préserver ses fonctions essentielles, de conserver et de 
cultiver des sols agricoles les plus riches possibles en matière organique pour éviter sa dégradation et assurer la sécurité alimentaire.

\section{Support de la production agricole}

Les sols marocains sont très variés du fait de la grande diversité des facteurs de pédogenèse du pays et des écosystèmes auxquels ils appartiennent. La formation des sols dépend des facteurs naturels tels que la géologie, la topographie, le climat et la végétation ainsi que de facteurs anthropiques (PNUE, 2003). La diversité du pays explique la présence de nombreux types de sols et un couvert végétal très hétérogène d'une région à une autre, variant des steppes sahariennes aux espaces forestiers relativement denses au Moyen Atlas. En outre, les sols portent l'empreinte d'une activité anthropique d'aménagement de l'espace (en particulier pour l'agriculture).

$\mathrm{Au}$ regard des conditions physiques et climatiques, les sols du Maroc sont une ressource fragile et sensible aussi bien aux aléas naturels qu'aux actions anthropiques d'autant plus que la majorité des terres agricoles se situe en milieu aride et semi-aride $(68 \%)$ caractérisé par des conditions climatiques sévères (Ministère délégué auprès du Ministre de l'énergie des mines, de l'eau et de l'environnement, 2016-2020).

Les terres agricoles au Maroc, représentent près de $69 \%$ du territoire, toutes fois une grande partie de cette surface est dédiée aux parcours (53 millions ha dont un tiers environ est surpâturé) et les forêts s'étendent sur 9 millions ha. Les terres cultivables ne couvrent ainsi que 8,8 millions ha (soit $18 \%$ de la superficie totale du pays). Environ 1,4 million ha, soit $15 \%$ de cette surface sont irrigables, le reste est constitué de terres bour10, avec $55 \%$ de la surface agricole utile (SAU) de bour défavorable et $27 \%$ en bour favorable (ENPARD, 2019). La question de l'eau est cruciale pour le développement de l'agriculture du pays.

Les plaines et collines à pluviométrie supérieure à $400 \mathrm{~mm}$ (le bour « favorable ») et les grands périmètres irrigués ont un potentiel de productivité relativement élevé. Ces régions ne représentent que le tiers de la SAU, le quart des exploitations et $64 \%$ du total irrigué du pays. Elles sont, en général, bien desservies en services et infrastructures socio-économiques et disposent des atouts pour être compétitives à l'international. Les zones plus difficiles (plaines et plateaux agricoles semi-arides, montagnes et steppes, zones oasiennes) regroupent les $3 / 4$ des exploitations, $2 / 3$ de la SAU et $36 \%$ de la SAU irriguée. Ces espaces d'agriculture traditionnelle correspondent en majorité à des zones à vocation pastorale ou agrosylvopastorale et à une irrigation de type traditionnel alimentée surtout par des eaux de surface. Ces zones subissent des contraintes multiples caractérisées notamment par de faibles disponibilités en ressources en eau et en sols (souvent dégradés); et une 
dotation insuffisante en équipements socio-économiques de base (routes, eau, électricité, etc.) (ENPARD, 2019).

Le régime alimentaire reste traditionnellement basé sur la consommation de céréales (blé, orge), d'agrumes (oranges, clémentines), d'olives, de rosacées fruitières (amandes, pommes, abricots...), de betteraves à sucre, de légumineuses alimentaires, de cultures maraichères dont les pommes de terre et les tomates. La production est extrêmement variable car elle est fortement liée à la pluviométrie ( Le conseil général du développement agricole, 2012). Cependant, la production nationale ne couvre pas les besoins du pays, même pendant les années humides de bonnes répartitions de précipitation.

Toutefois, la principale contrainte de l'agriculture marocaine est la forte dépendance aux aléas climatiques et notamment à une pluviométrie très erratique. Or les phénomènes de sécheresse observés en longue période semblent gagner en fréquence et en intensité et les écoulements des eaux de surface ont baissé de $35 \%$ en 30 ans. L'accès à l'eau avec seulement $1 / 8^{\text {ème }}$ de la SAU en irrigué est donc un élément majeur de la politique agricole marocaine.

\section{Soumission aux différentes influences externes}

$\mathrm{Au}$ Maroc, les sols sont caractérisés par une fragilité et une sensibilité aussi bien aux aléas naturels (érosion, sécheresse), qu'aux actions anthropiques (défrichements, surpâturage, arrachage des espèces ligneuses, urbanisation, etc.), ce qui entraine leur dégradation.

Cette dégradation touche les terres en sec (bour) et les terres irriguées. Il se traduit non seulement par une perte de production agricole, mais aussi par une perte de la biodiversité, une baisse de la séquestration du carbone, l'envasement des barrages et d'autres impacts (Jorio, 2017).

Toutefois, le processus de dégradation des sols le plus actif est celui de l'érosion hydrique qui constitue la principale menace environnementale à l'égard du capital sol. Ceci, est dû au climat marocain caractérisé par des pluies d'hiver et parfois des averses d'été très fortes qui provoquent des ruissellements importants entrainant une érosion significative. Les sols touchés par l'érosion hydrique moyenne à forte couvrent une superficie de plus de 12 millions d'hectares, soit $18,5 \%$ de la superficie totale du territoire national. Ce problème d'érosion engendre une perte de capacité de stockage des barrages par envasement de l'ordre de 75 millions $\mathrm{m}^{3} /$ an et une perte totale cumulée de 1750 millions $\mathrm{m}^{3}$ sur 17,5 milliards $\mathrm{m}^{3}$ de capacité totale de stockage des barrages (Le conseil général du développement agricole, 2012).

Par ailleurs, l'urbanisation impact négativement les terres agricoles. Le Maroc connait depuis un siècle un développement urbain rapide. Ce processus d'urbanisation continu qui s'est déclenché avec la mise en place du protectorat 
en 1912 et a poursuivi à ce jour, a complètement reconfiguré le territoire national et l'occupation de l'espace. Ainsi, le Maroc est passé d'une population à majorité rurale à une population à majorité urbaine, avec un taux d'urbanisation de $60 \%$. Ce qui pose la problématique de la capacité du royaume à contenir et affronter cette explosion urbaine (Badraoui, 2006). cette urbanisation galopante se fait de manière anarchique. Malheureusement, l'extension des villes se fait au dépend des terres de bonne qualité pour l'agriculture. Si rien n'est fait, le taux moyen de consommation des terres agricoles par l'urbanisation se maintient à 3500 ha/an, et l'étendue des terres consommées arrivera à 70000 ha à 1 'horizon 2025, soit $0,8 \%$ de la SAU. Cette réduction des terres agricoles pourrait présenter un enjeu alimentaire pour le pays (Badraoui, 2006).

De plus, les zones irriguées au Maroc sont touchées par la salinisation qui contribué à la dégradation des sols en ces zones. Elle est dû à plusieurs causes, telles que la surexploitation des nappes, notamment les nappes côtières; la remontée des nappes résultant de l'abus de l'eau d'irrigation; le mauvais drainage des sols dû au manque d'entretien des réseaux du drainage et la non-maitrise des techniques d'irrigation, notamment l'utilisation de l'irrigation gravitaire.Cette salinisation des sols affecte près de 500 à 160000 ha, soit environ 16\% des terres irriguées (Badraoui, 2006).

La dégradation des sols menace de plus en plus la sécurité alimentaire et les services écosystémiques au Maroc, et aggravée par les effets du changement climatique, elle constitue l'un des plus grands défis que le Maroc doit relever. Le secteur agricole du fait de sa place prépondérante dans l'utilisation des ressources hydriques sera le secteur le plus impacté par la baisse prévue des précipitations, tant pour les cultures pluviales que les cultures irriguées. Il sera aussi affecté par la hausse prévue des températures et par les effets en termes d'érosion (Le conseil général du développement agricole, 2012).

Ainsi, la durée de la «période de croissance », qui correspond au moment de la saison agricole pendant laquelle les conditions de précipitations et de température sont suffisantes pour permettre une croissance et un développement optimaux des cultures, devrait diminuer de 30 jours en 2050 par rapport à 2010. Cela signifie que la période de croissance actuelle de la plupart des céréales de novembre à avril sera réduite de novembre à mars d'ici à 2050 ce qui aura certainement un impact significatif sur les rendements (Badraoui et Balaghi, 2012).

Ainsi, les impacts du changement climatique devraient se traduire par une réduction de la superficie des cultures pluviales annuelles dans les plaines atlantiques. Badraoui et Balaghi (2012) indiquent que «les résultats des études d'impact du changement climatique sur l'agriculture marocaine réalisées par 1'Organisation des Nations Unies pour l'alimentation et 
l'agriculture (FAO), l'Institut National de la Recherche Agronomique (INRA) et la Direction de la Météorologie Nationale (DMN), prévoient une diminution de la productivité des principales cultures pluviales dans les zones semi-arides. Badraoui et Balaghi, 2012) montrent également que le changement climatique aura des conséquences négatives sur l'agriculture, notamment:

- La chute de la productivité agricole, surtout pour les cultures pluviales et celles pour lesquelles le progrès technologique réalisé est faible ou nul, tels que l'orge, le blé dur, le blé tendre, l'olivier, et les légumineuses alimentaires ;

- Le déplacement de l'aire de culture de certaines espèces comme celles qui sont exigeantes en eau (maïs, tournesol, fève, olivier) ou qui seront affectées par la hausse des températures (espèces arboricoles fruitières ayant besoin en froid) ;

- La perte de fertilité des sols en raison de la baisse de la matière organique des sols et de l'érosion hydrique et éolienne, et la baisse des disponibilités en eau d'irrigation.

Certes, le Maroc a adopté plusieurs stratégies économiques et agronomiques en vue de protéger et conserver ses ressources naturelles, toutefois, elles ne sont pas d'ailleurs les seules qui peuvent apporter des solutions adéquates au problème d'insécurité alimentaire. le droit peut également contribuer à empêcher l'abus de ces ressources (Motib, 2019).

\section{Vers une garantie de la sécurité alimentaire durable basée sur la préservation juridique des ressources naturelles}

L'évaluation des ressources naturelles alimentaires s'est basée dans cette recherche sur une approche méthodologique internationalement reconnue (PER): «Pression- Etat-Réponse », afin d'identifier les causes et les effets des problèmes environnementaux ainsi que les solutions concrètes à apporter. Le PER a montré que le Maroc dispose des atouts importants, mais, il est porteur de plusieurs sensibilités. Le diagnostic a fait montrer que les ressources naturelles subissent plusieurs pressions, qui contribuent à leur détérioration. Les "réponses" que nous adopterons ici, seront basées sur « la protection juridique » de ces ressources, pour atténuer les impacts sur le support naturel alimentaire. Il a été souligné que la «protection» ne signifie pas la non-utilisation des ressources alimentaires, mais plutôt leur utilisation durable, de telle sorte qu'elles profitent aux générations présentes sans compromettre les besoins des générations futures.

Il est vrai que les lois ne sont pas là pour donner directement à manger aux individus, mais elles créent les conditions propices en vue d'atteindre l'objectif de la sécurité alimentaire. En clair, les lois ne peuvent pas, à elles 
seules, prétendre être en mesure de résoudre les problèmes de la faim. Toutefois, elles peuvent contribuer à empêcher l'abus des ressources en eau, la rationalisation et l'efficience de l'utilisation de ces eaux et la lutte contre la pollution, et l'accaparement des terres productives. Elles peuvent créer les conditions propices pour nourrir une population et aussi doter les habitants d'outils légaux pour revendiquer leur accès aux ressources alimentaires. En effet, la sécurité alimentaire tire sa dimension juridique par rapport à son lien étroit avec le droit à une nourriture suffisante (Ziegler, 2003).

\section{Protection quantitative et qualitative des ressources en eau}

La question des ressources en eau au Maroc, apparait dans un aspect quantitatif, celui du manque d'eau, et qualitatif, celui de la pollution de l'eau. Les deux aspects de la question sont intimement liés et nécessitant une gestion et protection rigoureuse.

Certes, « l'eau se dégrade toujours par l'usage qui en fait. Si même la quantité restituée au système général hydrographique ne diminue pas toujours considérablement, c'est la qualité qui est atteinte, et par la suite la quantité restant encore disponible » (Waline, 1976), L'aspect qualitatif est par conséquent, intimement lié à l'aspect quantitatif et participe de ce mouvement de raréfaction de la ressource en eau, et plus précisément, de l'eau potable.

Sur le plan réglementaire, les ressources en eau sont actuellement régies par la loi 36-15 relative à l'eau (Dahir, 2016) qui a mis en place un certain nombre d'outils et des principes de protection de l'eau, en l'occurrence le principe de la domanialité publique des eaux, Ceux de l'interdiction et de l'autorisation préalable et celui de la répression. Cette réglementation générale apparaît comme le moyen le plus sûr pour protéger l'eau, car elle détermine clairement les objectifs à atteindre et les moyens à les employer.

Concernant la domanialité publique des eaux, la loi 36-15 relative à l'eau confirme ce principe, reprenant pour l'essentiel les dispositions introduites par les législations qui l'ont précédée par les Dahir de 1914, 1919 et la loi 95-10 (Dahir, 1995) relative à l'eau. La nouvelle loi considère l'eau comme un bien public ne pouvant faire l'objet d'appropriation privée sous réserve de situations qu'elle définit elle-même. Ainsi, le domaine public hydraulique peut faire l'objet d'usages à caractère privatifs qui ont pour effet de soustraire la ressource en eau aux règles de la domanialité publique du fait des droits acquis ou en vertu de demandes de particuliers à l'Administration d'une autorisation de prise d'eau; d'un règlement d'eau ou d'une concession de prise d'eau en contrepartie du paiement d'une redevance.

Par ailleurs, l'utilisation et l'exercice des activités dans les eaux se fait au moyen d'un principe général suivant lequel toute activité est interdite sauf autorisation. Deux types d'autorisation sont prévus par la loi 36-15 relative à l'eau : les autorisations destinées à se procurer de l'eau (Dahir 2016, article 
28) et les autorisations de déversement (Dahir 2016, article 98). Les autorisations relatives au domaine public hydraulique, sont accordées, en fonction de la disponibilité des ressources du domaine public hydraulique, après enquête publique.

Plus loin, à travers les dispositions du droit de l'eau, le législateur marocain, semble vouloir garantir une protection des eaux soit à usage agricole, ou à usage alimentaire, à travers un ensemble de conditions et des système d'interdiction (dahir 2016, article 49), de suivi, et de contrôle qui montre sa volonté à mettre contribution le droit à l'effet de préserver les ressources naturelles alimentaires pour la population.

Par ailleurs, si le diagnostic a montré que le Maroc est vulnérable aux changements climatiques, cela nécessite d'activer le rôle du Conseil supérieur de l'eau et du climat, qui a resté extrêmement absent depuis longtemps. Ainsi, pour revitaliser ce conseil et lui permettre de jouer un rôle plus actuel dans la résolution des problèmes et défis liés aux ressources en eau et au climat, la loi 36-15 relative à l'eau a élargi les compétences de ce conseil et l'a chargé, d'examiner et de donner son avis sur les orientations générales de la politique nationale en matière d'eau et du climat, notamment, la stratégie nationale d'amélioration de la connaissance du climat et de ses changements, des impacts de ces derniers sur les ressources en eau et des risques liés à l'eau, et le plan national de l'eau. II peut, également, donner son avis sur toute question relative à l'eau et au climat soumise à son examen par l'administration (dahir 2016, art 78).

Le Maroc n'est pas à l'abri, non plus, des phénomènes météorologiques extrêmes, comme les inondations. Au cours de la dernière décennie, il a connu plusieurs inondations sous forme de crues éclairs ou crues massives (Messouli et al, 2014). Dans ce contexte, la loi 36-15 relative à l'eau a mis en place un cadre institutionnel et des mécanismes pour la protection contre ces phénomènes, par l'élaboration des Plans de Gestion de Sécheresses aux niveaux des Agences des bassins hydrauliques qui doivent caractériser et surveiller les sécheresses et suivre les situations hydriques; et par la création de systèmes informatiques de suivi au niveau local et national. D'autres instruments sont aussi mis en disposition comme le « Atlas des Régions menacées d'inondation » (Dahir 2016, art 118), qui classera les régions selon le niveau de danger, et l'installation de systèmes d'alerte intégrée au niveau des oueds menaçants.

En vue de protéger les ressources en eau contre les effets négatifs de la pollution et de toute forme d'exploitation illicite des eaux souterraines et superficielles, la loi $\mathrm{n}^{\circ} 36-15$ relative à l'eau a accordé au contrôle du domaine public hydraulique une importance particulière, confiant cette mission à la police des eaux. Ce dispositif ne date pas d'aujourd'hui, elle est plutôt prévue par la loi relative à l'eau émise en 1995 (Dahir, 1995). Comme elle est prévu 
par d'autres textes relatifs à l'eau depuis 1925 durant le protectorat, néanmoins n'a jamais été vraiment opérationnelle à cause d'un manque de moyens humains, matériels et judiciaires adaptés aux infractions.

La création de la police des eaux est principalement liée à la nécessité de garantir pour tous le droit d'accès à l'eau et d'assurer également la bonne gouvernance dans la gestion des ressources en eau, en veillant aux respect des principes de participation, de concertation, de gestion intégrée et décentralisée ainsi que la consolidation de la solidarité spatiale et de la protection du milieu naturel, tout en visant la consécration de l'exploitation durable des ressources en eau (La nouvelle Tribune, 2017). Ce dispositif vient donc à point nommé pour contrôler l'utilisation de cette richesse qui se fait de plus en plus rare, à travers l'ouverture des enquêtes et des poursuites judiciaires contre les auteurs impliqués dans les actes d'exploitation illégale de ces ressources. La loi 36-15 relative à l'eau considère comme contraventions toute utilisation ou exploitation du domaine public hydraulique sans autorisation ou concession préalable prévues respectivement dans les articles 28 et 33 du la loi susmentionné (Dahir, 2016).

\section{Légaliser l'accès à la terre comme un outil de conservation des sols}

La terre cultivable se présente comme l'un des éléments de base pour assurer la sécurité alimentaire d'une population. D'abord, il faut que la terre serve prioritairement à produire des denrées alimentaires plutôt que des végétaux non alimentaires. Et il faut aussi qu'elle soit le plus possible réservée à l'agriculture et qu'elles puissent maintenir dans la durée. Mais avant tout cela il faut préserver les sols fertiles (Olivier, 2014).

Sur le plan réglementaire, le sol est actuellement régi par le Dahir $\mathrm{n}^{\circ} 1$ 69-170 du 25 juillet 1969 sur la défense et la restauration des sols (Dahir, 1969). A citer également la loi n¹2-90 relative à l'urbanisme qui comporte plusieurs dispositions de protection des terres agricoles et des forêts contre des usages abusifs pour l'urbanisation, comme l'article 4 de la loi susmentionnée qui précise que le schéma directeur d'aménagement urbain détermine les zones d'urbanisation et les dates à compter desquelles elles peuvent être ouvertes à l'urbanisation en préservant notamment les terres agricoles et les zones forestières dont les limites sont fixées par voie réglementaire.

Par ailleurs, l'article 19 définit les objectifs environnementaux du plan d'aménagement Ainsi, il est noté que ce document a pour objectif de définir l'affectation des différentes zones suivant l'usage principal qui doit en être fait ou la nature des activités dominantes qui peuvent y être exercées telles que zone d'habitat, commerciale, touristique, maraîchère, agricole, forestière, et les zones dans lesquelles toute construction est interdite. L'article susmentionné prévoit aussi les règles d'utilisation des sols (Nejjari et al., 2014). 
La loi $\mathrm{n}^{\circ} 11-03$ relative à la protection et à la mise en valeur de l'environnement (Dahir, 2013) est également un grand acquis en faveur de la protection et la mise en valeur des ressources en sols, elle dispose que le sol, le sous-sol et les richesses qu'ils contiennent, en ressources limitées ou non renouvelable, sont protégés contre toute forme de dégradation et doivent être exploités de manière rationnelle. Il prévoit notamment que des mesures particulières de protection sont édictées afin de lutter contre la désertification, les inondations, la disparition des forêts, l'érosion, les pertes de terres arables et la pollution du sol et de ses ressources, dus notamment à l'utilisation des produits et pesticides chimiques. Lesdites mesures peuvent être déclarées d'utilité publique et s'imposer à tout exploitant ou bénéficiaire ( Dahir 2003, art 18).

D’autre part, l'article 18 de la loi suscitée apporte un élément important en la matière. Elle prévoit que l'affectation et l'aménagement du sol à des fins agricoles, industrielles, minières, touristiques, commerciales, urbaines, ainsi que les travaux de recherche archéologique ou d'exploitation des ressources du sous-sol susceptibles de porter atteinte à l'environnement, sont soumis à autorisation préalable suivant les cas et conformément aux conditions fixées par les textes législatifs et réglementaires (dahir 3003, art18).

Une loi spécifique au sol est en train d'être élaborée et vise la mise en valeur et la protection du sol contre diverses formes de dégradation et de pollution. L'initiative pour la préparation de cette loi est prise conformément aux directives contenues dans la loi $n^{\circ} 11-03$ relative à la protection et à la mise en valeur de l'environnement. La loi inclut un certain nombre d'instruments connexes concernant : La gestion rationnelle de l'utilisation des sols, Les aspects spécifiques à la pollution des sols, La réhabilitation des sites contaminés, La responsabilité pour des atteintes aux sols

\section{Conclusion}

Les ressources naturelles ont permis à certains pays de se situer sur le sentier du développement durable. Si l'on veut répondre aux besoins d'une population qui ne cesse de s'accroitre et assurer la sécurité alimentaire. Il faut préserver, reconstituer ces ressources. Cette étude a permis de réaffirmer que le Maroc, regorge de beaucoup de potentialités distinguées. En effet, les ressources naturelles sont une excellente source d'aliments et constituent un moteur de création d'emplois. Malheureusement, suite à la pression anthropique et à l'exploitation anarchique qui pèsent sur ces ressources, ces derniers risquent de se dégrader si rien n'est fait dans un proche avenir. Leur épuisement est devenu l'une des questions les plus préoccupantes pour l'avenir du pays.

Toutefois, les différents textes de loi analysés prévoient des dispositions permettant d'infléchir les pratiques anthropiques qui pèsent sur 
les ressources naturelles en consacrant des aspects de conservation et de gestion.

\section{References:}

1. Abdellah Laouina, 2016: gestion durable des ressources naturelles et de la biodiversité au Maroc, prospective « Maroc 2030 », rapport pour le compte de haute commissariat- au plan, Royaume du Maroc, Mars, p 8 et 43 .

2. Abdellatif Khattabi et Lelia Croitoru, 2017 : Eau, Le Coût de La dégradation de L'environnement au MAROC, World Bank group report number 105633-ma Banque mondiale, Janvier, p 14.

3. Abdeljaouad Jorio, 2017 : La Dégradation des sols : Concept Et approche, le Coût de La dégradation de L'environnement au MAROC, Banque mondiale, p 49.

4. A.Nejjari. A.Ejkoufi. Kamal Targuisti, 2014: les lois de l'urbanisme au service de l'environnement au Maroc, Revista de Estudios Andaluces $\mathrm{N}^{\mathrm{o}} 31$, p 38.

5. Brun-Roudier Jean, 2004 : Mise en place d'un outil informatique d'aide à la gestion intégrée du bassin de l'Huisne et élaboration d'indicateurs de suivi, septembre, Rapport, p 49.

6. Cédric Le Guillou, 2011 : Effets combinés de la qualité des résidus de culture et de la disponibilité en azote minéral sur la stabilisation de la structure du sol par les microorganismes, thèse en sciences de l'environnement, Université Européenne de Bretagne, p 7.

7. Gourou Pierre, 1940, L'utilisation du sol en Indochine française. Paris: Paul Hartmann, P 466.

8. Ibtissam Motib, 2019 : protection juridique et gestion durable des ressources naturelles au Maroc : cas des communes littorales du Gharb, entre Sidi Taibi et Mnasra (province de kenitra), thèse nationale en Géographie Physique et Environnementale, universite Ibn Tofail, Faculté des lettres et des sciences humaines, Kenitra, p 159-160.

9. Mohamed Badraoui, 2006 : Connaissance et utilisation des ressources en sol au Maroc, 25/01/06, p 107-108.

10. Mohamed Badraoui et Riad Balaghi, 2012 : l'adaptation de l'agriculture marocaine au changement climatique, Environnement et Changement Climatique au Maroc, Diagnostic et Perspectives, publié par Konrad-Adenauer-Stiftung, p 62-63.

11. Mohammed Messouli et al, 2014 : anticipation et gestion des risques d'évènements climatiques extrêmes et de catastrophes naturelles au Maroc, Rapport de synthèse, Institut Royal des études stratégiques, Décembre, p 34. 
12. Olivier, U. D. (2014). La problématique juridique de la sécurité alimentaire en Haïti de 2002 à 2012 au regard du droit à l'alimentation, Édition numérique réalisée le 23 novembre 2016 à Chicoutimi, Québec, p 17.

13. Requier-Desjardins Mélanie, 2012 : Enjeux et modes d'intégration de la dimension socio-économique dans la surveillance environnementale, Options méditerranéennes, B 68, p 181.

14. Samir Benmalek, 2017 : changements climatiques, ces dangers qui pèsent sur les ressources hydriques du Maroc, le Matin, mise en ligne le 16 octobre 2017. P 6. https://lematin.ma/journal/2017.

15. Samir Benmalek, 2018 : Ressources hydriques : Le rapport annuel « Solutions basées sur la nature pour l'eau » publié aujourd'hui par l'Unesco, mises en ligne 18/03/2018. P. 7.

16. WALINE .M, 1976 « hypothèses sur l'évolution du droit en fonction de la raréfaction de certains », revue de droit prospectif, $n^{\circ}$ 2, P. 12.

17. Jean Ziegler, 2003 : Le droit à l'alimentation. Mille et une nuits, 2003, p17-18.

18. ENPARD Méditerranée, 2019 : Rapport de synthèse sur l'agriculture au Maroc, Janvier, p 27- 28.

19. FAO, 1996 : Production alimentaire: le rôle déterminant de l'eau, sommet Mondiale de l'alimentation, 13-17 Novembre 1996, Rome Italie, documents d'information technique. http://www.fao.org/3/w2612f/w2612f07a.htm.

20. Haut-Commissariat au Plan, 2018: Prospective Maroc 2030, quelle démographie, $\mathrm{p} 6$.

21. Haut Commissariat au Plan, 2006 : statistiques environnementales au MAROC, le plan bleu, Eurostat, p 63.

22. HLPE, (2012). Sécurité alimentaire et changement climatique, Rapport du Groupe d'experts de haut niveau sur la sécurité alimentaire et la nutrition du Comité de la sécurité alimentaire mondiale, Rome. $13 p$.

23. L'Institut royal des études stratégiques, 2011 : le Maroc face au changement climatique Incidences sur la trajectoire de développement et perspectives d'adaptation, Rapport stratégique de synthèse, Février, p 8.

24. Le conseil général du développement agricole, 2012 : situation de l'agriculture marocaine, Décembre, $\mathrm{N}^{\circ} 10 \mathrm{p} 24$ et 66.

25. Le Matin en partenariat avec le Secrétariat d'état auprès du Ministre de l'équipement, du transport, de la logistique et de l'eau chargé de l'eau et Lydec, 2017 : Problématique de l'eau au Maroc, l'heure est à l'action, Changements climatiques, Ces dangers qui pèsent sur les ressources hydriques du Maroc,16 Octobre, P 6. 
26. La nouvelle Tribune société, 2017 : La Police des Eaux entre en action, mise en ligne le 20 décembre, p 1. https://lnt.ma/police-eaux-entreaction/.

27. Ministère de l'énergie, des mines et de l'environnement, département de l'environnement, 2016 Stratégie nationale de l'eau, p 31-32.

28. Ministère délégué auprès du Ministre de l'Energie, des Mines, de l'Eau et de 1'Environnement, chargé de l'Environnement, 2014 : Politique du changement climatique au MAROC, Royaume du MAROC, Mars, p 14.

29. Ministère délégué auprès du Ministre de l'Energie, des Mines, de l'Eau et de l'Environnement, chargé de l'Environnement, 2014: politique du changement climatique au Maroc, Vers un développement faiblement carboné et résilient au changement climatique, Mars, p 17.

30. Ministère délégué auprès du Ministre de l'énergie des mines, de l'eau et de l'environnement, 2016 : Stratégie et plan d'actions national pour la diversité biologique du Maroc, 2016-2020, P 20.

31. Ministère Délégué auprès du Ministère de l'Energie, de l'Eau et de l'Environnement, Chargé de l'Eau, 2015 : état de la qualité des ressources en eau au Maroc, p 3.

32. Ministère délégué auprès du ministère de l'énergie, des mines de l'eau et de l'environnement chargé de l'environnement, Plan cadre national pour la consommation et production durables,2015, rapport Switch Med : Maroc/2015, p 32.

33. PNUE plan bleu, 2003 : Les menaces sur les sols dans les pays méditerranéens, étude bibliographique, rapport préparé par Laura de Franchis (Plan Bleu) avec la collaboration de François Ibanez (Plan Bleu) pour la cartographie, Mai, p 3-4.

34. Seconde communication nationale, 2016 : Réalités des changements climatique au Maroc, rapport compilation de la SCNCC, prévention WEB, p 11.

35. RBF, 2019 : évaluation environnementale \& sociale stratégique, programme axe sur les résultats pour l'amélioration de la compétitivité territoriale au Maroc (paract-m), PARACT-M, Octobre, p 47.

36. World Trade Organization 2010, World Trade Report : Trade in naturalresources, p. 46.

37. World Bank, 2017 : Gestion de la Rareté d'Eau Milieu Urbain Maroc, public disclosure authorized, Rapport, Novembre, Royaume du Maroc, $\mathrm{P} 12$.

38. WRI,2014: World Resources Institute, Aqueduct's Global Water Risk Mapping, p 14. 
39. Dahir $\mathrm{n}^{\circ} 1-16-113$ du 6 Kaada 1473 (10 aout 2016) pourtant promulgation de la loi 36-15 relative à l'eau, B.O n 65064 moharem $1438, \mathrm{p} 1482$.

40. Dahir $\mathrm{n}^{\circ} 1-95-154$ du 18 rabii I 1416 (16 août 1995) portant promulgation de la loi ${ }^{\circ} 10-95$ sur l'eau, Bulletin officiel $n^{\circ} 4325 \mathrm{du}$ 24 rabii 1416 (20 septembre 1995).

41. Dahir $\mathrm{n}^{\circ}$ 1-69-170 du 10 joumada I 1389 (25 juillet 1969) sur la défense et la restauration des sols. B. O nº : 2960-bis du 29/07/1969, $\mathrm{P}, 802$.

42. Dahir $n^{\circ} 1-03-59$ du 10 rebii I 1424 (12 mai 2003) portant promulgation de la loi $\mathrm{n}^{\circ} 11-03$ relative à la protection et à la mise en valeur de l'environnement, B.O du 10 Rebii I 1424; p 500. 